\title{
High Doses of Dexamethasone Induce Endoplasmic Reticulum Stress-Mediated Apoptosis by Promoting Calcium Ion Influx-Dependent CHOP Expression in Osteoblasts
}

\author{
Yunshan Guo ( $\nabla$ 183869503@qq.com ) \\ Xi'an Jiaotong University https://orcid.org/0000-0001-6671-3300 \\ Dingjun Hao \\ Xi'an Jiaotong University
}

\section{Research Article}

Keywords: osteoporosis, dexamethasone, apoptosis, endoplasmic reticulum stress, CHOP, osteoblasts

Posted Date: July 30th, 2021

DOI: https://doi.org/10.21203/rs.3.rs-723110/v1

License: (c) (1) This work is licensed under a Creative Commons Attribution 4.0 International License. Read Full License

Version of Record: A version of this preprint was published at Molecular Biology Reports on October 26th, 2021. See the published version at https://doi.org/10.1007/s11033-021-06806-y. 


\section{Abstract}

Background: The molecular mechanisms by which dexamethasone (Dex) induces apoptosis in osteoblasts remain unclear.

Materials and Methods: MC3T3-E1 cells were treated with $0,10^{-8}, 10^{-6}$, and $10^{-4} \mathrm{M}$ Dex for $24 \mathrm{~h}$. The expression of ATF6, and phosphorylated PERK and IRE1, cell apoptosis, and the activity of caspase-12 and caspase- 3 were measured. The expression of $\mathrm{CHOP}$ and the rate of influx of calcium ions were also measured in cells treated with 0 and $10^{-4} \mathrm{M}$ Dex for $24 \mathrm{~h}$. The effect of 2-APB treatment was assessed in cells treated with 0 or $10^{-4} \mathrm{M}$ Dex.

Results: The levels of ATF6 and phosphorylated PERK and IRE1 increased in a dose-dependent manner in MC3T3-E1 cells treated with $10^{-8}, 10^{-6}$, and $10^{-4} \mathrm{M}$ Dex, compared to in cells treated with $0 \mathrm{M}$ Dex $(\mathrm{P}$ $<0.05$ ). Cells treated with $10^{-6}$ and $10^{-4} \mathrm{M}$ Dex had significantly increased cell apoptosis rates and caspase-12 and caspase-3 activity compared to the control $(\mathrm{P}<0.05)$. Cells treated with $10^{-4} \mathrm{M}$ Dex had significantly increased levels of CHOP and calcium ion influx rates compared to in the control $(P<0.05)$. Combined treatment with $10^{-4} \mathrm{M}$ Dex and 2-APB abrogated the observed increases in cell apoptosis and the activity of caspase-12 and caspase-3 $(P>0.05)$.

Conclusion: High doses of Dex induce endoplasmic reticulum stress-mediated apoptosis by promoting calcium ion influx-dependent expression of $\mathrm{CHOP}$, and the activation of caspase- 12 and caspase- 3 in osteoblasts. Combined treatment with 2-APB protects the cells from the effects of Dex, preventing endoplasmic reticulum stress-mediated apoptosis.

\section{Introduction}

Dexamethasone (Dex) is an anti-inflammatory and immunosuppressive drug that is widely used in the treatment of rheumatoid arthritis, inflammatory bowel disease, chronic obstructive pulmonary disease, and other diseases. High doses of Dex may cause glucocorticoid-induced osteoporosis (GIOP)[1-3]. In patients receiving Dex, $30-50 \%$ of patients experience a fracture, with a $75 \%$ increased risk of fracture before a significant decrease in bone mineral density (BMD) occurs within the first 3 months of Dex treatment. The decrease in BMD reaches its peak at 3 to 6 months after the initiation of Dex treatment, and then continues to decrease steadily over time[4-6]. The rapid decrease in BMD observed during the early stages of Dex treatment is caused by an increase in osteoclast activity, while the slower decrease in BMD over the long term is related to a decrease in the activity of osteoblasts[7-8].

Osteoblasts play an important role in ensuring the normal bone mass of the body by forming bone tissue. Osteoporosis is a systemic metabolic change characterized by low bone mass, which can lead to increased bone fragility and increase the risk of fracture[9-10]. Recent studies have shown that the occurrence of osteoporosis is related to endoplasmic reticulum (ER) stress[11]. The ER is an important membranous organelle that is responsible for protein synthesis and folding, regulating the balance of 
calcium ions inside and outside the cell, and calcium storage. When intracellular calcium ion homeostasis is unbalanced, and unfolded or misfolded proteins accumulate in the ER cavity, the intracellular environment is affected and the ER loses its normal physiological function, leading to a series of adaptive regulatory processes termed ER stress[12-13].

Different intensities of ER stress have a bidirectional regulatory effect on osteoblast function. Under low levels of ER stress, the expression of Runx2 and other bone forming factors in osteoblasts increases, which promote the formation and reconstruction of bone tissue and effectively alleviate the progression of osteoporosis[14]. However, under excessive ER stress, the synthesis of ATF4 and ATF3 increases, promoting the expression of CHOP. High levels of CHOP activate the apoptotic pathway and accelerates the apoptosis of osteoblasts, leading to osteoporosis[15-16]. Although both Dex and ER stress induce osteoporosis by mediating osteoblast apoptosis, whether Dex leads to osteoblast apoptosis by inducing osteoblast ER stress is not clear.

In the present study, we explored the effect of Dex on ER stress in an osteoblast cell line to identify the mechanisms by which Dex induces osteoporosis. The effects of 2-APB, which can modify calcium channels, on osteoblast cells receiving Dex was assessed in parallel to determine if calcium antagonists can improve Dex-induced osteoporosis. Our findings could provide new targets for the treatment of iatrogenic osteoporosis caused by high doses of Dex.

\section{Materials And Methods}

\section{Cell culture}

Mouse calvaria osteoblasts, MC3T3-E1 cells, were purchased from Cell Resource Center (Shanghai Institute for Biological Sciences, Chinese Academy of Sciences). MC3T3-E1 cells were cultured in aminimum essential medium containing $10 \% \mathrm{FBS}, 100 \mathrm{U} / \mathrm{mL}$ penicillin, and $100 \mathrm{~g} / \mathrm{mL}$ streptomycin under $5 \% \mathrm{CO}_{2}$ in an incubator at a constant temperature of $37^{\circ} \mathrm{C}$. When the cells reached $80-90 \%$ fusion, cells in the logarithmic growth phase were selected for experimentation.

\section{Cell transfection}

Cells were seeded in 6-well plates at a density of $2 \times 10^{5} /$ well. CHOP siRNA and control siRNA were transfected using Lipofectamine 2000+ Opti Mem I liposome according to the manufacturer's instructions. The CHOP siRNA sequence was 5- GCGCATG $\forall G G A G \forall A G \forall C A G G$ - 3, while the control siRNA sequence was 5- T CTC $\forall C T G T G A C G T G T$ - 3 . After transfection for $24 \mathrm{~h}$, the culture medium was changed and cells were cultured in an incubator at $37^{\circ} \mathrm{C}$ under $5 \% \mathrm{CO}_{2}$.

\section{Western blot}

After cells were lysed, the cell lysates was quantified using a BCA assay, diluted to the same concentration, and heated at $95^{\circ} \mathrm{C}$ for 8 min with $4 \times$ sample loading buffer. Proteins were separated on 
an $8 \%$ acrylamide gel electrophoretically by SDS-PAGE, then transferred onto a PVDF membrane and blocked with 5\% skim milk. Primary antibodies were diluted with TBST as follows: ATF6 (1:1000), phosphorylated PERK (1:500), PERK (1:800), phosphorylated IRE1 (1:500), IRE1 (1:800), CHOP (1:500), and GAPDH (1:1000). The PVDF membrane was incubated overnight in diluted primary antibodies at $4^{\circ} \mathrm{C}$, and then incubated at room temperature in the dark for $1 \mathrm{~h}$ in horseradish peroxidase-labeled secondary antibodies, diluted at 1:20000 in TBST. Finally, protein bands were detected using the Odyssey two-color infrared laser imaging system.

\section{TUNEL staining}

Cells in suspension were added to the inner cover glass of a cell culture plate and cultured overnight as before. Cells were fixed with $4 \%$ paraformaldehyde at room temperature for $10 \mathrm{~min}$. Sheep serum was dropped onto the cover glass in 50 ul aliquots and incubated for 20 min at room temperature. After washing with PBS, 50 ul of anti-TUNEL reaction mixture was added, and cells were incubated at $37^{\circ} \mathrm{C}$ for 60 min. After washing with PBS, 50 ul of DAPI (at 1:100 dilution) was added and incubated at room temperature for $8 \mathrm{~min}$. Cover glasses were covered with $50 \%$ glycerin solution. The proportion of TUNEL positive cells was determined using an inverted fluorescence microscope.

\section{Caspase -12 and caspase -3 activity assays}

To measure caspase- 12 activity, cells were removed from plates by digesting with trypsin, collected in a centrifuge tube and counted, and about $5 \times 10^{4}$ cells were removed and centrifuged. The supernatant was discarded, and 50 ul of cell lysis buffer was added, and the cells were lysed in an ice bath for 15 min. Reaction buffer ( $50 \mathrm{ul}$ ) was added to each tube and mixed on a vortex, and then $5 \mathrm{ul}$ of ATAD-AFC was added to each tube, mixed, and incubated at $37^{\circ} \mathrm{C}$ for $90 \mathrm{~min}$. Aliquots of each reaction mixture (100 ul) were transferred to a 96-well plate and the absorbance was measured with an excitation wavelength of $400 \mathrm{~nm}$ and an absorption wavelength of $505 \mathrm{~nm}$ on a microplate reader.

Caspase- 3 activity was measured similarly, except that $2 \times 10^{5}$ cells were lysed in 100 ul of lysis buffer, and $10 \mathrm{ul}$ of cell lysate was combined with $10 \mathrm{ul}$ of AC-DEVD-PNA and $80 \mathrm{ul}$ of detection buffer in wells of a 96 well plate. The absorbance was also measured on a microplate reader, with an excitation wavelength of $400 \mathrm{~nm}$ and an absorption wavelength of $505 \mathrm{~nm}$.

\section{Calcium ion influx detection}

Cells were cultured overnight as before and allowed to adhere, and were then were washed twice with PBS. After the supernatant was discarded, 5mM Fluo8-AM solution was added to the plate and incubated for $60 \mathrm{~min}$ in the dark at room temperature. The supernatant was removed and discarded, and 4mM TG solution was added to the plate and incubated for $40 \mathrm{~min}$ in the dark at room temperature. After the supernatant was discarded, cells were washed twice in calcium ion-free PBS. The fluorescent signal was measured using a confocal laser microscope. The emission wavelength was $510 \mathrm{~nm}$, and the excitation wavelengths were $380 \mathrm{~nm} / 340 \mathrm{~nm}$. After the initial fluorescence reading was obtained for $30 \mathrm{~s}, 2 \mathrm{mM}$ 
$\mathrm{CaCl}_{2}$ solution was added to each well and the fluorescent signal was measured again to determine the rate of influx of calcium ions.

\section{Real-time PCR}

Total RNA was extracted using the TRIzol reagent, and reverse transcription was performed according to the manufacturer's instructions using the SYBR Prime Scripttm RT-PCR Kit II (Takara Bio, Japan). GAPDH was used as the reference gene. The forward primer for CHOP was 5

- GACGC Т CACTACTC Т GAC CTGCG - 3, and the reverse primer was 5 - GGATGTGCGTGTGAC TCTGT - 3. The forward primer for GAPDH was 5 - CGCTCTCTGCTC TC TG T - 3, and the reverse primer was 5- $\mathbb{C} A T G G T G T C T G A G C G A T G T$ - 3. The PCR conditions were as follows: pre-denaturation at $95^{\circ} \mathrm{C}$ for $5 \mathrm{~min}$, followed by 37 cycles of $94^{\circ} \mathrm{C}$ denaturation for $20 \mathrm{~s}$, annealing at $59^{\circ} \mathrm{C}$ for $20 \mathrm{~s}$, and elongation at $72^{\circ} \mathrm{C}$ for $20 \mathrm{~s}$. Ct values were used to quantify the relative transcription levels of CHOP mRNA. The mRNA relative expression level was calculated using the $2^{-\triangle \Delta \mathrm{Ct}}$ method.

\section{Statistical analysis}

All statistical analysis was performed using SPSS 22.0 statistical software (IBM, USA). All experiments were independently replicated 3-6 times, and data are expressed as the mean \pm standard deviation. The data from each group was normally distributed and had homogenous variance. One-way ANOVA was used for comparisons between multiple groups, and a LSD-t test was used for pairwise comparisons. A P $<0.05$ was considered to be statistically significant.

\section{Results}

\section{High doses of Dex induce ER stress-mediated apoptosis in osteoblasts}

ER stress-induced osteoblasts apoptosis is one of the pathogenic features of osteoporosis[11]. To determine whether Dex participates in the regulation of ER stress-mediated apoptosis in osteoblasts, mouse osteoblastic MC3T3-E1 cells were treated with different doses of Dex $\left(0,10^{-8}, 10^{-6}\right.$, and $\left.10^{-4} \mathrm{M}\right)$ for 24 hours. The expression of ATF6, phosphorylated PERK, and phosphorylated IRE1 were measured by western blot. Compared with the control group, cells receiving Dex responded with dose-dependent increases in the levels of ATF6, phosphorylated PERK, and phosphorylated IRE1 ( $P<0.05$, Figure 1a). Different doses of Dex may therefore induce ER stress in osteoblasts. TUNEL staining was used to confirm that Dex can induce ER stress-mediated osteoblast apoptosis. A dose of $10^{-8} \mathrm{M}$ Dex had no effect on apoptosis in osteoblasts, compared to the control group $(P>0.05$, Figure $1 b)$. Conversely, doses of $10^{-6}$ and $10^{-4} \mathrm{M}$ Dex both significantly increased the rate of apoptosis in osteoblasts compared to that of the control $\left(P<0.05\right.$, Figure $1 \mathrm{~b}$ ). Next, caspase-12 and caspase-3 activity were measured, and $10^{-8} \mathrm{M}$ Dex had no effect on their activity compared to in the control $(P>0.05$, Figure $1 \mathrm{c}$ and $1 \mathrm{~d})$. Accordingly, $10^{-}$ ${ }^{6}$ and $10^{-4} \mathrm{M}$ Dex significantly increased the activity of caspase-12 and caspase -3 in osteoblasts, 
compared to in the control $(P<0.05$, Figure $1 \mathrm{c}$ and $1 \mathrm{~d})$. These results suggest that different

concentrations of Dex can induce ER stress in osteoblasts, with higher concentrations $\left(10^{-6}\right.$ and $\left.10^{-4} \mathrm{M}\right)$ inducing ER stress-mediated apoptosis in osteoblasts.

\section{High doses of Dex lead to ER stress-mediated apoptosis by inducing CHOP expression in osteoblasts}

To further clarify the mechanism by which high doses of Dex regulate ER stress-mediated apoptosis in osteoblasts, MC3T3-E1 cells were treated with 0 or $10^{-4} \mathrm{M}$ Dex, and CHOP expression was detected by western blot. Compared with the control group, the expression of $\mathrm{CHOP}$ was significantly increased in cells receiving $10^{-4} \mathrm{M}$ Dex $(\mathrm{P}<0.05$, Figure $2 \mathrm{a})$. To further confirm the effects of $\mathrm{CHOP}$ on Dex regulation of ER stress-mediated apoptosis in osteoblasts, CHOP siRNA was transfected into MC3T3-E1 cells. We found that CHOP expression was significantly decreased in MC3T3-E1 cells after transfection with CHOP siRNA $(P<0.05$, Figure $2 b)$. MC3T3-E1 cells were transfected with CHOP siRNA or control siRNA, then treated with 0 or $10^{-4} \mathrm{M}$ Dex. In the Control siRNA group, $10^{-4} \mathrm{M}$ Dex significantly increased the rate of apoptosis of MC3T3-E1 cells compared with $0 \mathrm{M}$ Dex $(P<0.05$, Figure $2 c)$. However, in the CHOP siRNA group, $10^{-4} \mathrm{M}$ Dex did not change the rate of apoptosis of MC3T3-E1 cells compared with $0 \mathrm{M}$ Dex (P $>0.05$, Figure $2 \mathrm{c}$ ). Caspase- 12 and caspase-3 activity assays further confirmed the results. In the control siRNA group, $10^{-4} \mathrm{M}$ Dex significantly increased the activity of caspase- 12 and caspase-3 compared with $0 \mathrm{M}$ Dex $\left(\mathrm{P}<0.05\right.$, Figure $2 \mathrm{~d}$ ). However, in the CHOP siRNA group, $10^{-4} \mathrm{M}$ Dex had no effect on caspase-12 and caspase- 3 activity compared with $0 \mathrm{M} \mathrm{Dex}(P>0.05$, Figure $2 \mathrm{~d})$. These results suggest that high doses of Dex induce ER stress-mediated apoptosis by promoting CHOP expression in osteoblasts. Moreover, CHOP expression is essential for high dose Dex-induced ER stress-mediated apoptosis in osteoblasts.

\section{High doses of Dex induce ER stress by promoting calcium influx in osteoblasts}

Calcium homeostasis imbalance induces ER stress[17-19]. To further clarify the mechanisms by which Dex induces ER stress in osteoblasts, MC3T3-E1 cells were treated with 0 or $10^{-4} \mathrm{M}$ Dex, and the calcium ion influx was measured. Compared with the control group, calcium ion influx was significantly increased in osteoblasts receiving $10^{-4} \mathrm{M} \operatorname{Dex}(\mathrm{P}<0.05$, Figure $3 \mathrm{a})$. This suggests that high doses of Dex promote calcium ion influx in MC3T3-E1 cells. To further confirm the role of calcium ion influx in Dex-induced ER stress in osteoblasts, cells were simultaneously treated with Dex and 2-aminoethoxydiphenyl borate (2APB), a blocker of store operated calcium influx. MC3T3-E1 cells were treated with 10mM 2-APB or DMSO, then treated with 0 or $10^{-4} \mathrm{M}$ Dex. The expression levels of ATF6, phosphorylated PERK, and phosphorylated IRE1 were subsequently measured as before. Cells receiving DMSO and $10^{-4} \mathrm{M}$ Dex had significantly increased levels of ATF6, phosphorylated PERK, and phosphorylated IRE1 compared with cells receiving DMSO and $0 \mathrm{M}$ Dex $(P<0.05$, Figure $3 \mathrm{~b})$. However, in cells receiving 2-APB, $10^{-4} \mathrm{M}$ Dex did not increase the levels of ATF6, phosphorylated PERK, or phosphorylated IRE1 compared with cells receiving $0 \mathrm{M}$ Dex $(P>0.05$, Figure $3 b)$. These results suggest that Dex induces ER stress by promoting calcium ion influx. The effect of calcium ion influx on ER stress was further verified by measuring the 
expression and mRNA transcription levels of CHOP. In the DMSO treatment group, $10^{-4} \mathrm{M}$ Dex significantly increased the expression and mRNA levels of CHOP compared with $0 \mathrm{M}$ Dex $(P<0.05$, Figure $3 \mathrm{c}$ and $3 \mathrm{~d}$ ). However, in the 2-APB treatment group, $10^{-4} \mathrm{M}$ Dex did not increase the expression and $\mathrm{mRNA}$ levels of CHOP compared with $0 \mathrm{M}$ Dex $(P>0.05$ and $P<0.05$, respectively, Figure $3 c$ and $3 d)$. These results suggest that high doses of Dex induce ER stress by promoting calcium ion influx.

\section{2-APB protects osteoblasts from Dex-induced ER stress-mediated apoptosis}

To further confirm the effect of 2-APB on Dex induction of ER stress-mediated apoptosis in osteoblasts, MC3T3-E1 cells were treated with 2-APB or DMSO, then with 0 or $10^{-4} \mathrm{M}$ Dex. Apoptosis was detected by TUNEL staining. In the DMSO group, $10^{-4} \mathrm{M}$ Dex significantly increased the apoptosis of MC3T3-E1 cells compared with $0 \mathrm{M}$ Dex $(\mathrm{P}<0.05$, Figure $4 \mathrm{a})$. However, in the 2-APB group, $10^{-4} \mathrm{M}$ Dex did not increase the apoptosis of MC3T3-E1 cells compared with 0 M Dex ( $>>0.05$, Figure 4a). Caspase-12 and caspase-3 activity assays further confirmed this result. In the DMSO treatment group, $10^{-4} \mathrm{M}$ Dex significantly increased the activity of caspase- 12 and caspase- 3 compared with $0 \mathrm{M} \operatorname{Dex}(\mathrm{P}<0.05$, Figure $4 \mathrm{~b}$ and $4 \mathrm{c})$. However, in the 2-APB treatment group, $10^{-4} \mathrm{M}$ Dex did not increase the activity of caspase-12 and caspase- 3 compared with $0 \mathrm{M}$ Dex $(P>0.05$, Figure $4 \mathrm{~b}$ and $4 \mathrm{c})$. These results suggest that 2-APB protects osteoblasts from Dex-induced ER stress-mediated apoptosis. Therefore, blocking calcium influx may be a new method to relieve Dex-induced osteoporosis.

\section{Discussion}

Osteoporosis is the most serious side effect of Dex treatment[1-3]. The apoptosis of osteoblasts induced by Dex is the main cause of osteoporosis caused by Dex. Maintaining the correct number of osteoblasts is essential for bone formation. Decreases in the number of osteoblasts caused by excessive apoptosis plays a role in the pathogenesis of osteoporosis[4-6]. The response of cells to ER stress, including apoptosis, can have far-reaching effects in the body, and ER stress-mediated apoptosis is important in many diseases, including osteoporosis[21-22].

Recent studies have shown that osteoblasts undergo ER stress during BMP-induced osteoblast differentiation. Some ER stress markers are also expressed and play an important role in osteoblast differentiation. OASIS is a key transcription factor of ER stress, which is involved in bone formation by promoting COL1A1 transcription and extracellular matrix secretion in osteoblasts[23]. XBP1, another important ER stress effector, can directly bind to the promoter region of Runx2, a key transcription factor for osteogenesis, thereby promoting osteogenesis[24]. ATF4, an important ER stress transcription factor, can promote collagen secretion and amino acid transport in osteoblasts[25-26]. Although a large number of studies have confirmed that many key molecules of ER stress can promote osteogenesis, some have also found that excessive ER stress can lead to apoptosis of osteoblasts. Under excessive ER stress, the synthesis of ATF4 and ATF3 increases, promoting the expression of CHOP. High levels of 
$\mathrm{CHOP}$ expression activate the apoptotic pathway, leading to apoptosis of osteoblasts and accelerating the progression of osteoporosis[27-29].

A variety of injury factors acting on the ER can activate ER stress by causing intracellular calcium ion imbalances or luminal misfolding, leading to the accumulation of unfolded proteins[30]. There are three ER stress-sensing proteins residing in the endoplasmic omentum: activating transcription factor 6 (ATF6), RNA-activated protein kinase pancreatic elf-2 kinase (PERK), and inositol requiring enzyme1 (IRE1). These proteins can bind GRP78/BIP to form a stable complex. In the absence of ER stress, these proteins are in a bound state and have no activity. When ER stress occurs, the accumulation of unfolded proteins allows GRP78/BIP to separate from the three transmembrane proteins. These three proteins are activated by dissociation from GRP78. IRE1 and PERK were activated by dimerization and autophosphorylation, respectively. ATF6 is transported from the endoplasmic omentum to the Golgi body, and then cleaved by a specific protease to form activated ATF6. Increased expression of ATF6, phosphorylated IRE1, and phosphorylated PERK is a marker of ER stress[31-33]. In the present study, the levels of ATF6, phosphorylated IRE1, and phosphorylated PERK in osteoblasts increased in a dose-dependent manner with $10^{-8}, 10^{-6}$ and $10^{-4} \mathrm{M}$ Dex, suggesting that Dex can induce ER stress in osteoblasts.

Caspases are the main effector molecules that mediate cell apoptosis. Caspase-12 is a specific molecule of ER stress-mediated apoptosis. Knockout of caspase-12 can significantly reduce ER stress-mediated apoptosis. Caspase-12 can mediate apoptosis through activation of cytochrome C-independent pathways, via caspase- 9 , caspase-7, and caspase-3[34-35]. Caspase- 3 is a common molecule in many pathways that mediate apoptosis[36-37]. In the present study, we found that $10^{-8} \mathrm{M}$ Dex had no effect on apoptosis and the activation of caspase- 12 and caspase-3, while $10^{-6} \mathrm{M}$ and $10^{-4} \mathrm{M}$ Dex significantly increased apoptosis and the activity of caspase-12 and caspase-3. High doses of Dex can therefore induce ER stress-mediated apoptosis in osteoblasts.

$\mathrm{CHOP}$ is a transcription factor that is specific to ER stress, which belongs to the transcription factor CCAAT/ enhancer binding protein (C/EBP) family, and often binds to other members of the transcription factor CCAAT/ enhancer binding protein (C/EBP) family, resulting in the formation of dimer[38]. Under normal conditions, CHOP is expressed at low levels, but upon ER stress, activation of IRE1, PERK, and ATF- 6 can promote the expression of CHOP. CHOP can induce apoptosis by promoting the expression of apoptotic genes such as Bax, GADD34, and ER01, but it can also induce apoptosis by inhibiting the expression of anti-apoptotic genes, such as Bcl-2[39-40]. In the present study, CHOP expression was significantly increased in osteoblasts after treatment with $10^{-4} \mathrm{M}$ Dex. When CHOP expression was inhibited by CHOP siRNA, the Dex-mediated effects on apoptosis and caspase activity were abrogated. These results suggest that high doses of Dex can affect ER stress-mediated apoptosis by promoting $\mathrm{CHOP}$ expression in osteoblasts. Moreover, CHOP expression is essential for Dex-induced ER stressmediated apoptosis in osteoblasts.

Calcium ions are important secondary messengers, and play an important role in various cellular physiological and pathological activities. Calcium ion homeostasis is the basis for maintaining the 
normal structure and function of cells. The ER is the main site for protein synthesis, secretion, modification, and transport in cells, and also the main organelle for maintaining intracellular calcium ion homeostasis[41-42]. The level of free calcium ions in the cytoplasm are much lower than in the ER under normal physiological conditions. The lyanodine receptor (RyR), the 1,4, 5-triphosphate receptor (IP3R), and the sarcoplasmic reticulum calcium pump are three calcium channels located in the endoplasmic reticulum, which are closely related to calcium ion release and calcium ion uptake. The dynamic homeostasis of intracellular calcium ions is maintained by the ER releasing calcium ions into the cytoplasm via RyR and IP3R, while calcium ions are taken up from the cytoplasm by calcium pumps into the ER[43-44]. Exogenous stimulation can induce changes in calcium channels on the endoplasmic omentum, causing calcium depletion or overload, thus disrupting the synthesis, folding, and modification of proteins in the ER. Misfolded protein accumulation in the in the ER causes ER stress[45-47]. In the present study, calcium ion influx was significantly increased in osteoblasts receiving $10^{-4} \mathrm{M}$ Dex, and 2APB, a blocker of store operated calcium influx. Accordingly, $10^{-4} \mathrm{M}$ Dex did not affect the levels of ATF6, phosphorylated PERK, and phosphorylated IRE1 after pretreatment with 2-APB. These results confirm that high doses of Dex induced ER stress by promoting calcium influx in osteoblasts. 2-APB similarly prevented the effects of Dex on CHOP expression, apoptosis, and the activation of caspase- 12 and caspase-3. These results suggest that high doses of Dex induce ER stress by promoting calcium ion influx. The application of 2-APB protects osteoblasts from Dex-induced ER stress-mediated apoptosis. However, the specific mechanisms by which Dex promotes calcium ion influx still need to be explored in detail.

\section{Conclusion}

In conclusion, in the present study we found that high doses of Dex induce ER stress-mediated apoptosis by increasing calcium ion influx, promoting CHOP expression, and activating caspase- 12 and caspase- 3 in osteoblasts. The application of 2-APB protects osteoblasts from Dex-induced ER stress-mediated apoptosis. Therefore, blocking calcium influx may be a new avenue for treating Dex-induced osteoporosis. These findings provide a theoretical basis for developing novel methods for clinical prevention of osteoporosis induced by Dex.

\section{Declarations}

Funding This study was supported by the Natural Science Foundation of Shannxi Province (2020JM687) and the Science and Technology Plan of Xi'an City (2020yb35).

Authors' contributions GYS performed the experiments, analysed the data and wrote the manuscript; HDJ designed the study, supervised the study and revised the manuscript.

Consent to Participate All authors have confirmed their participation in this study.

Consent to Publish All authors have agreed to publish this manuscript. 


\section{Compliance with ethical standards}

Conflict of interest The authors declare that they have no confict of interest.

Ethics approval The study protocol was approved by the Ethics Committee of Hong Hui Hospital, Xi'an Jiao Tong University (No. 202103023). All procedures performed in studies were in accordance with the ethical standards of the institutional and/or national research committee.

\section{Informed consent None.}

\section{Acknowledgements}

We would like to thank Editage (www.editage.cn) for English language editing.

\section{References}

1. Lane NE. Glucocorticoid-Induced Osteoporosis: New Insights into the Pathophysiology and Treatments. Curr Osteoporos Rep. 2019;17(1):1-7.

2. Canalis E, Mazziotti G, Giustina A, Bilezikian JP. Glucocorticoid-induced osteoporosis: pathophysiology and therapy. Osteoporos Int. 2007;18(10):1319-28.

3. Chotiyarnwong P, McCloskey EV. Pathogenesis of glucocorticoid-induced osteoporosis and options for treatment. Nat Rev Endocrinol. 2020;16(8):437-447.

4. Saito Y, Nakamura S, Chinen N, Shimazawa M, Hara H. Effects of anti-osteoporosis drugs against dexamethasone-induced osteoporosis-like phenotype using a zebrafish scale-regeneration model. J Pharmacol Sci. 2020;143(2):117-121.

5. Weinstein RS. Glucocorticoid-induced osteonecrosis. Endocrine. 2012;41(2):183-90.

6. $\mathrm{Hu} \mathrm{H}, \mathrm{Li} \mathrm{Z}$, Lu M, et al. Osteoactivin inhibits dexamethasone-induced osteoporosis through upregulating integrin beta1 and activate ERK pathway. Biomed Pharmacother. 2018;105:66-72.

7. Deng S, Dai G, Chen S, et al. Dexamethasone induces osteoblast apoptosis through ROSPI3K/AKT/GSK3beta signaling pathway. Biomed Pharmacother. 2019;110:602-608.

8. Zhu CY, Yao C, Zhu LQ, She C, Zhou XZ. Dexamethasone-induced cytotoxicity in human osteoblasts is associated with circular RNA HIPK3 downregulation. Biochem Biophys Res Commun. 2019;516(3):645-652.

9. Hadjidakis DJ, Androulakis II. Bone remodeling. Ann N Y Acad Sci. 2006;1092:385-96.

10. Lee WC, Guntur AR, Long F, Rosen CJ. Energy Metabolism of the Osteoblast: Implications for Osteoporosis. Endocr Rev. 2017;38(3):255-266.

11. Li J, Yang S, Li X, et al. Role of endoplasmic reticulum stress in disuse osteoporosis. Bone. 2017;97:2-14.

12. Mahdi AA, Rizvi SH, Parveen A. Role of Endoplasmic Reticulum Stress and Unfolded Protein Responses in Health and Diseases. Indian J Clin Biochem. 2016;31(2):127-37. 
13. Oakes SA, Papa FR. The role of endoplasmic reticulum stress in human pathology. Annu Rev Pathol. 2015;10:173-94.

14. Chen Y, Guo Y, Li J, et al. Endoplasmic reticulum stress remodels alveolar bone formation after tooth extraction. J Cell Mol Med. 2020;24(21):12411-12420.

15. Hamamura K,Yokota $\mathrm{H}$. Stress to endoplasmic reticulum of mouse osteoblasts induces apoptosis and transcriptional activation for bone remodeling. FEBS Lett. 2007,581(9): 1769-1774.

16. Park SJ,Kim KJ,Kim WU,et al. Involvement of endoplasmic reticulum stress in homocysteine-induced apoptosis of osteoblastic cells. Bone Miner Metab,2012;30(4): 474-484.

17. Mekahli D, Bultynck G, Parys JB, De Smedt H, Missiaen L. Endoplasmic-reticulum calcium depletion and disease. Cold Spring Harb Perspect Biol. 2011;3(6):a004317.

18. Nakanishi K, Kakiguchi K, Yonemura S, Nakano A, Morishima N. Transient Ca2+ depletion from the endoplasmic reticulum is critical for skeletal myoblast differentiation. FASEB J. 2015;29(5):2137-49.

19. Luciani DS, Gwiazda KS, Yang TL, et al. Roles of IP3R and RyR Ca2+ channels in endoplasmic reticulum stress and beta-cell death.Diabetes. 2009;58(2):422-32.

20. Bootman MD, Collins TJ, Mackenzie L, Roderick HL, Berridge MJ, Peppiatt CM. 2aminoethoxydiphenyl borate (2-APB) is a reliable blocker of store-operated Ca2+ entry but an inconsistent inhibitor of InsP3-induced Ca2+ release. FASEB J. 2002;16(10):1145-50.

21. Park SJ, Kim KJ, Kim WU, Oh IH, Cho CS. Involvement of endoplasmic reticulum stress in homocysteine-induced apoptosis of osteoblastic cells. J Bone Miner Metab. 2012;30(4):474-84.

22. Tang $\mathrm{CH}$, Chiu YC, Huang CF, Chen YW, Chen PC. Arsenic induces cell apoptosis in cultured osteoblasts through endoplasmic reticulum stress. Toxicol Appl Pharmacol. 2009;241(2):173-81

23. Murakami T, Saito A, Hino S, et al. Signalling mediated by the endoplasmic reticulum stress transducer OASIS is involved in bone formation. Nat Cell Biol. 2009;11(10):1205-11.

24. Liu Y, Zhou J, Zhao W, et al. XBP1S associates with RUNX2 and regulates chondrocyte hypertrophy. J Biol Chem. 2012;287(41):34500-13.

25. Zhang K, Wang M, Li Y, et al. The PERK-EIF2alpha-ATF4 signaling branch regulates osteoblast differentiation and proliferation by PTH. Am J Physiol Endocrinol Metab. 2019;316(4):E590-E604.

26. Yang S, Hu L, Wang C, Wei F. PERK-elF2alpha-ATF4 signaling contributes to osteogenic differentiation of periodontal ligament stem cells. J Mol Histol. 2020;51(2):125-135.

27. Sano R, Reed JC. ER stress-induced cell death mechanisms. Biochim Biophys Acta. 2013;1833(12):3460-3470.

28. lurlaro R, Muñoz-Pinedo C. Cell death induced by endoplasmic reticulum stress. FEBS J. 2016;283(14):2640-52.

29. Liu L, Zhang Y, Gu H, Zhang K, Ma L. Fluorosis induces endoplasmic reticulum stress and apoptosis in osteoblasts in vivo. Biol Trace Elem Res. 2015;164(1):64-71.

30. Saito A, Imaizumi K. Endoplasmic reticulum stress response in osteogenesis. Clin Calcium. 2013;23(11):1569-75. 
31. Hetz C, Papa FR. The Unfolded Protein Response and Cell Fate Control. Mol Cell. 2018;69(2):169-181.

32. Kaneko M, Imaizumi K, Saito A, et al. ER Stress and Disease: Toward Prevention and Treatment. Biol Pharm Bull. 2017;40(9):1337-1343.

33. Gardner BM, Pincus D, Gotthardt K, Gallagher CM, Walter P. Endoplasmic reticulum stress sensing in the unfolded protein response. Cold Spring Harb Perspect Biol. 2013;5(3):a013169.

34. Szegezdi E, Fitzgerald U, Samali A. Caspase-12 and ER-stress-mediated apoptosis: the story so far. Ann N Y Acad Sci. 2003;1010:186-94.

35. García de la Cadena S, Massieu L. Caspases and their role in inflammation and ischemic neuronal death. Focus on caspase-12. Apoptosis. 2016;21(7):763-77.

36. Porter AG, Jänicke RU. Emerging roles of caspase-3 in apoptosis. Cell Death Differ. 1999;6(2):99-104.

37. Liu J, Mao J, Jiang Y, et al. AGEs Induce Apoptosis in Rat Osteoblast Cells by Activating the Caspase3 Signaling Pathway Under a High-Glucose Environment In Vitro. Appl Biochem Biotechnol. 2016;178(5):1015-27.

38. Oyadomari S, Mori M. Roles of CHOP/GADD153 in endoplasmic reticulum stress. Cell Death Differ. 2004;11(4):381-9.

39. Hu H, Tian M, Ding C, Yu S. The C/EBP Homologous Protein (CHOP) Transcription Factor Functions in Endoplasmic Reticulum Stress-Induced Apoptosis and Microbial Infection. Front Immunol. 2019;9:3083.

40. Liu W, Zhu X, Wang Q, Wang L. Hyperglycemia induces endoplasmic reticulum stress-dependent CHOP expression in osteoblasts. Exp Ther Med. 2013;5(5):1289-1292.

41. Krebs J, Agellon LB, Michalak M. Ca(2+) homeostasis and endoplasmic reticulum (ER) stress: An integrated view of calcium signaling. Biochem Biophys Res Commun. 2015;460(1):114-21.

42. Krebs J, Groenendyk J, Michalak M. Ca2+-signaling, alternative splicing and endoplasmic reticulum stress responses. Neurochem Res. 2011;36(7):1198-211.

43. Luciani DS, Gwiazda KS, Yang TL, et al. Roles of IP3R and RyR Ca2+ channels in endoplasmic reticulum stress and beta-cell death. Diabetes. 2009;58(2):422-32.

44. Santulli G, Nakashima R, Yuan Q, Marks AR. Intracellular calcium release channels: an update. J Physiol. 2017;595(10):3041-3051.

45. Yu L, Jiang B, Chen Z, et al. Cytisine induces endoplasmic reticulum stress caused by calcium overload in HepG2 cells.Oncol Rep. 2018;39(3):1475-1484.

46. Hamamura K, Yokota H. Stress to endoplasmic reticulum of mouse osteoblasts induces apoptosis and transcriptional activation for bone remodeling. FEBS Lett. 2007;581(9):1769-74.

47. Wang M, Kaufman RJ. Protein misfolding in the endoplasmic reticulum as a conduit to human disease. Nature. 2016;529(7586):326-35.

\section{Figures}


Figure 1

a
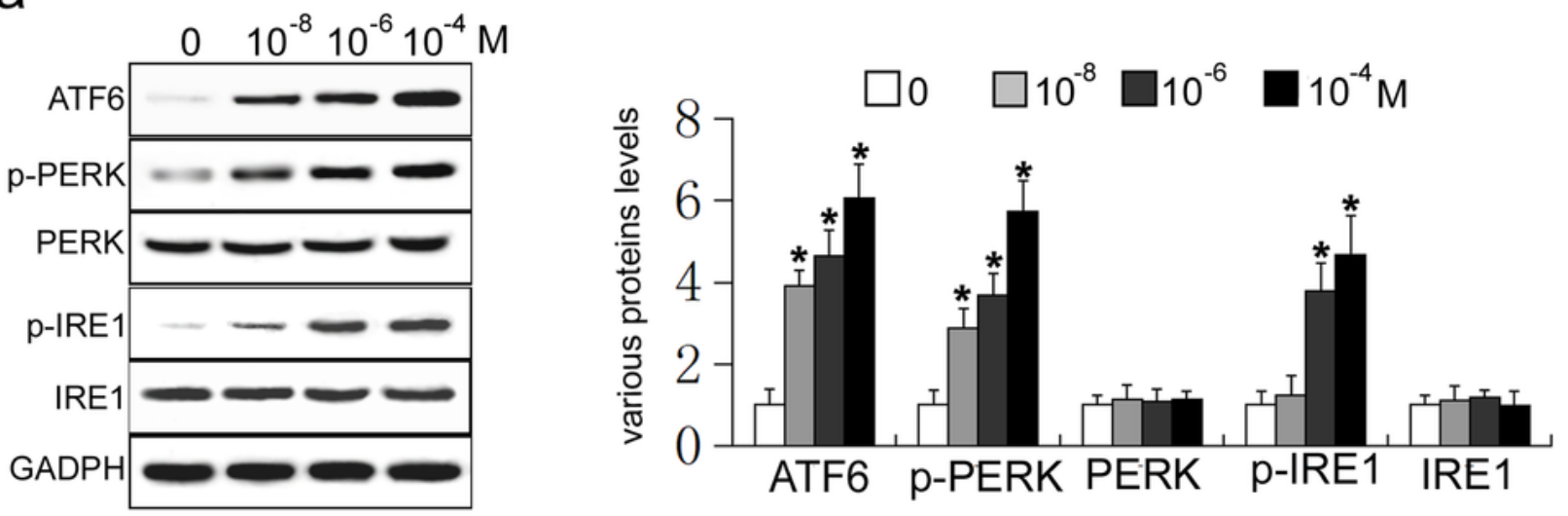

b
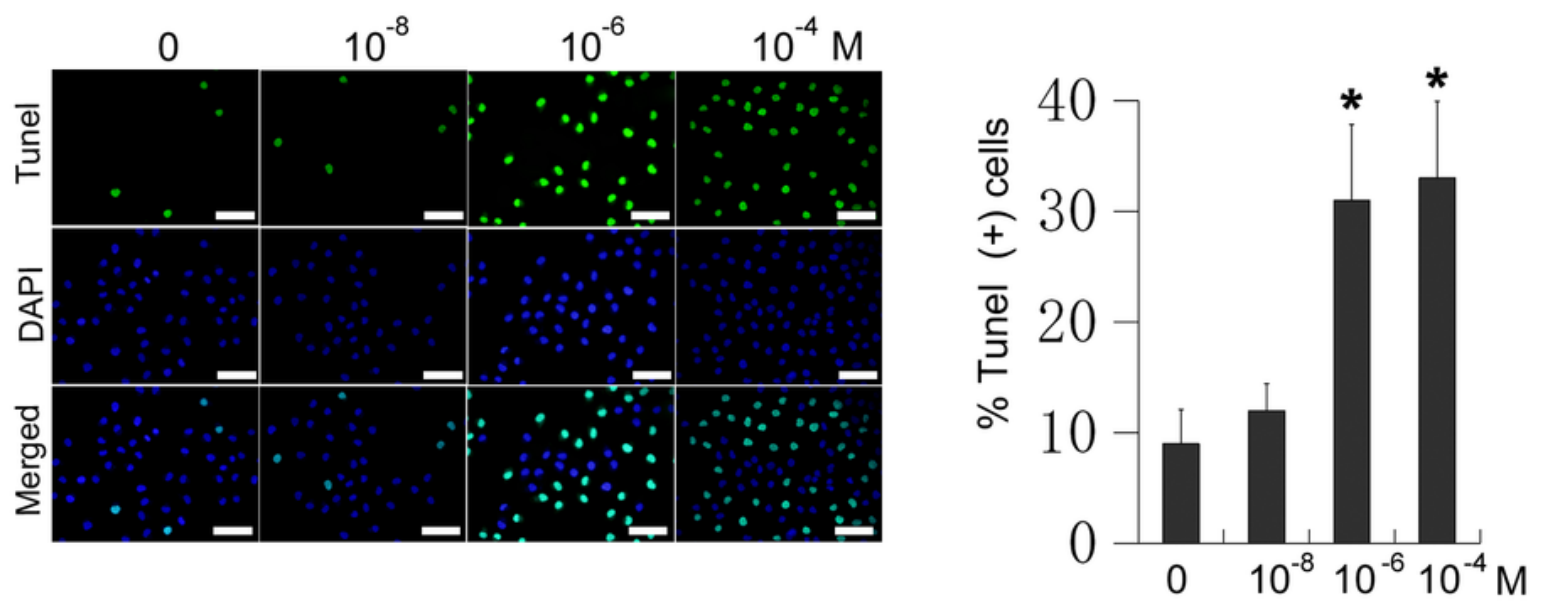

C

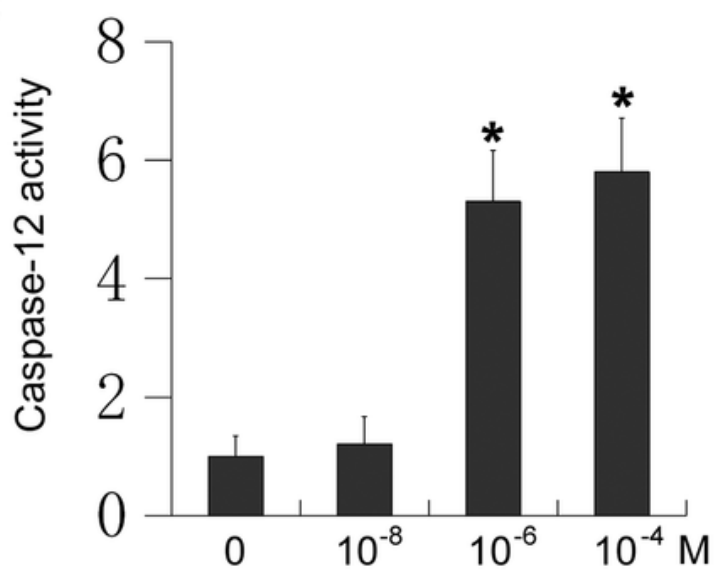

d

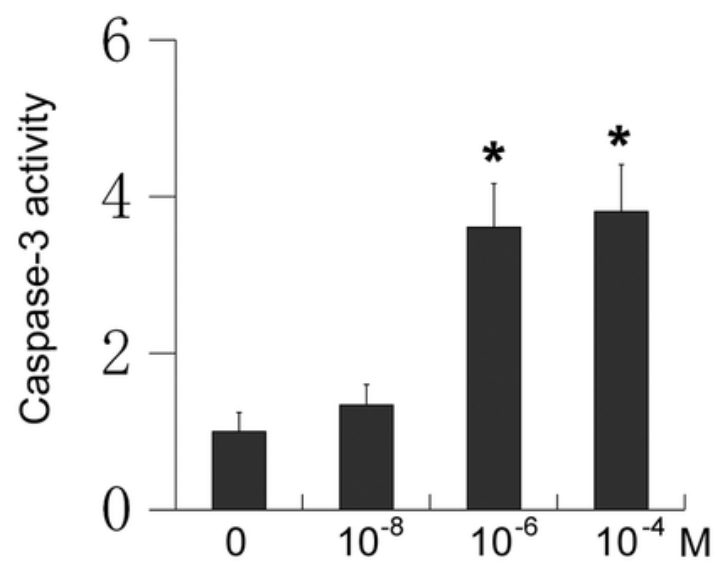

Figure 1

MC3T3-E1 cells were treated with Dex (0, 10-8, 10-6, and 10-4 M) for $24 \mathrm{~h}$. a. Western blot of ATF6, phosphorylated PERK (p-PERK), PERK, phosphorylated IRE1 (p-IRE1), and IRE1 in MC3T3-E1 cells. b. TUNEL staining was used to detect the effect of different concentrations of Dex on apoptosis in MC3T3E1 cells $($ Scale $=50 \mu \mathrm{m}) ;$ c. Caspase-12 activity was measured in cells receiving Dex; D. Caspase-3 activity was measured in cells receiving Dex. 
Figure 2

a
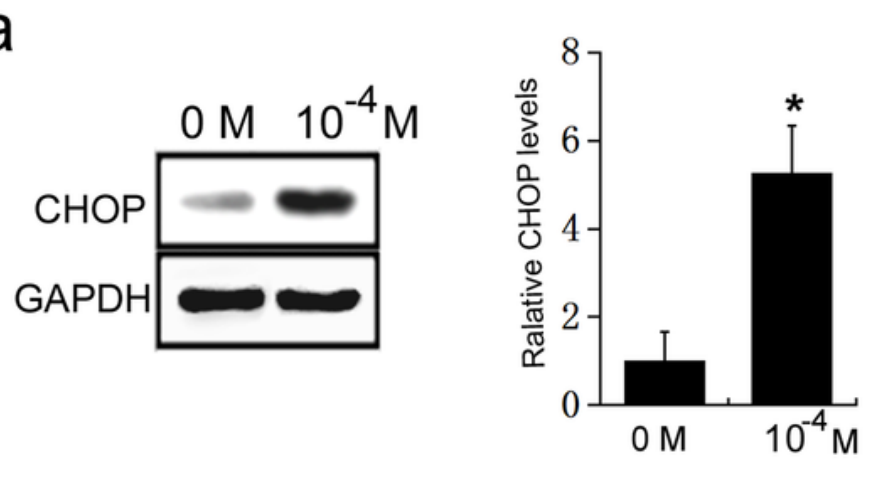

C

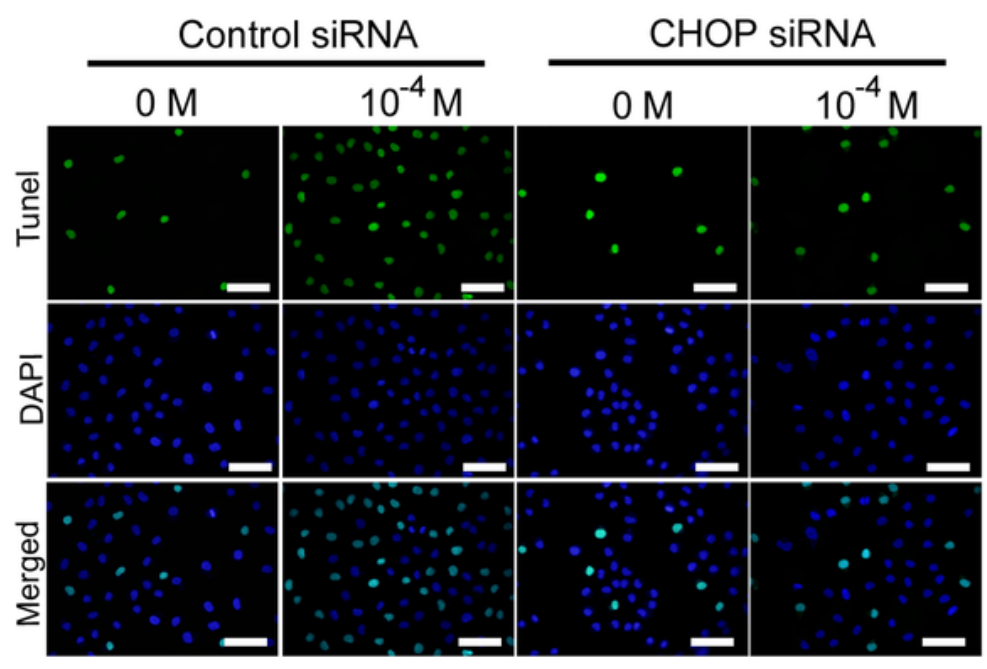

d

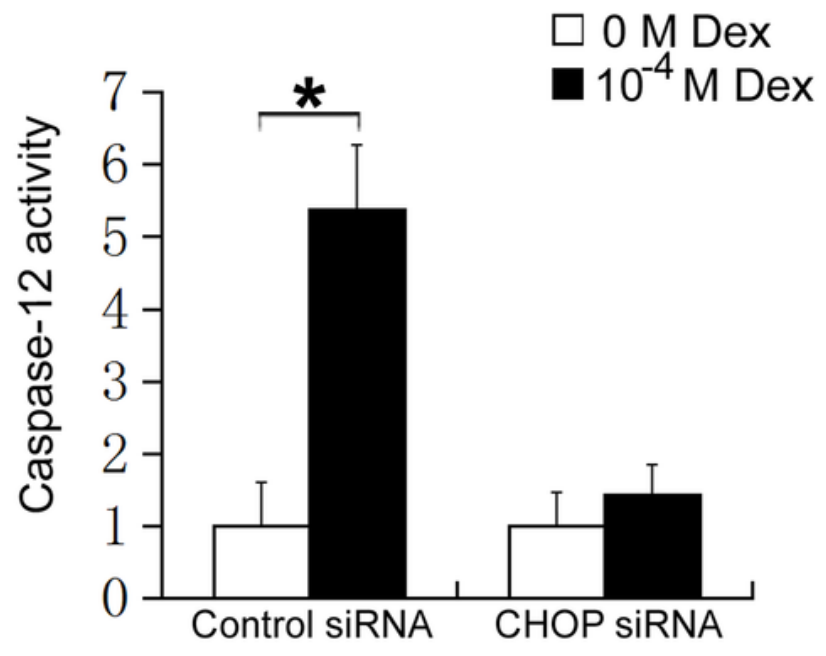

b

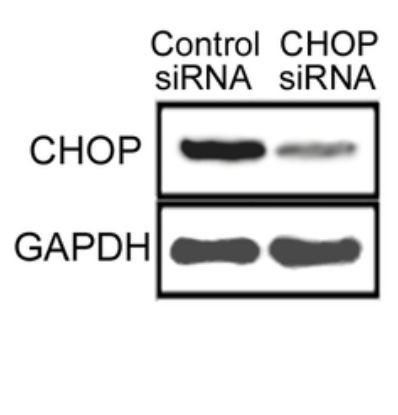

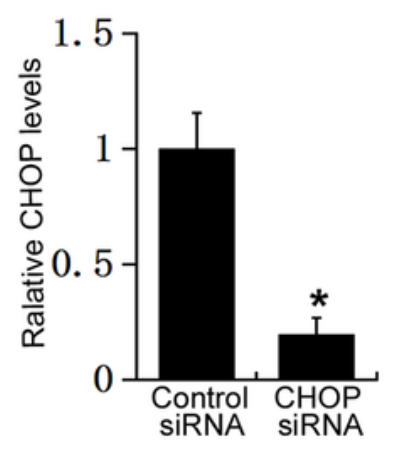
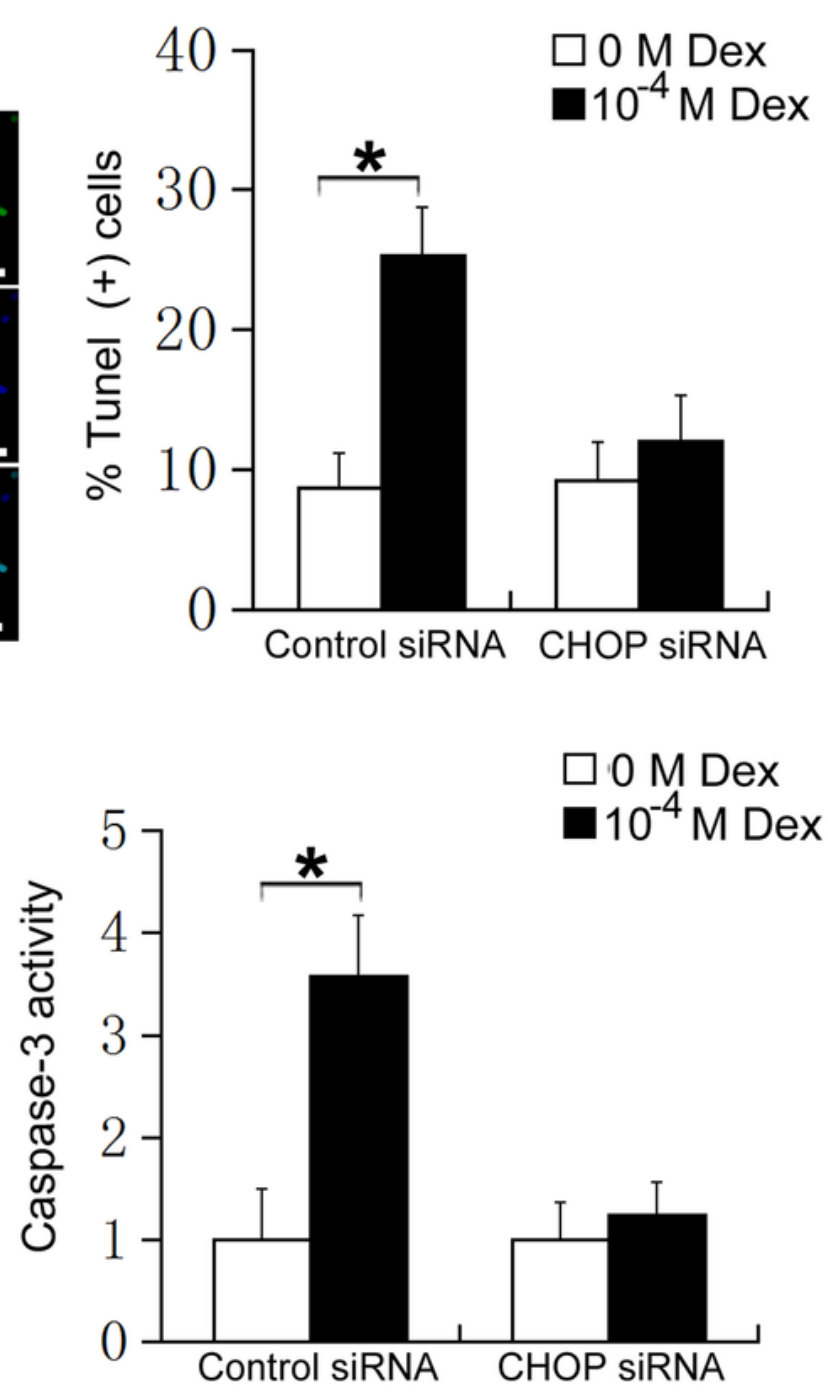

Figure 2

a. MC3T3-E1 cells were treated with 0 or 10-4 M Dex, and the expression of CHOP was measured by western blot; b. MC3T3-E1 cells were transfected with control siRNA or CHOP siRNA, and CHOP expression was detected by western blot. MC3T3-E1 cells were transfected with control siRNA or CHOP siRNA, and then treated with 0 or 10-4 M Dex for $24 \mathrm{~h}$; c. TUNEL staining was used to detect apoptosis in

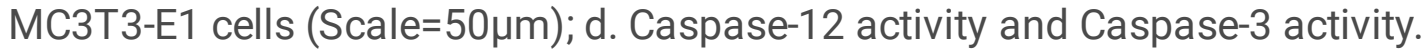


Figure 3

a
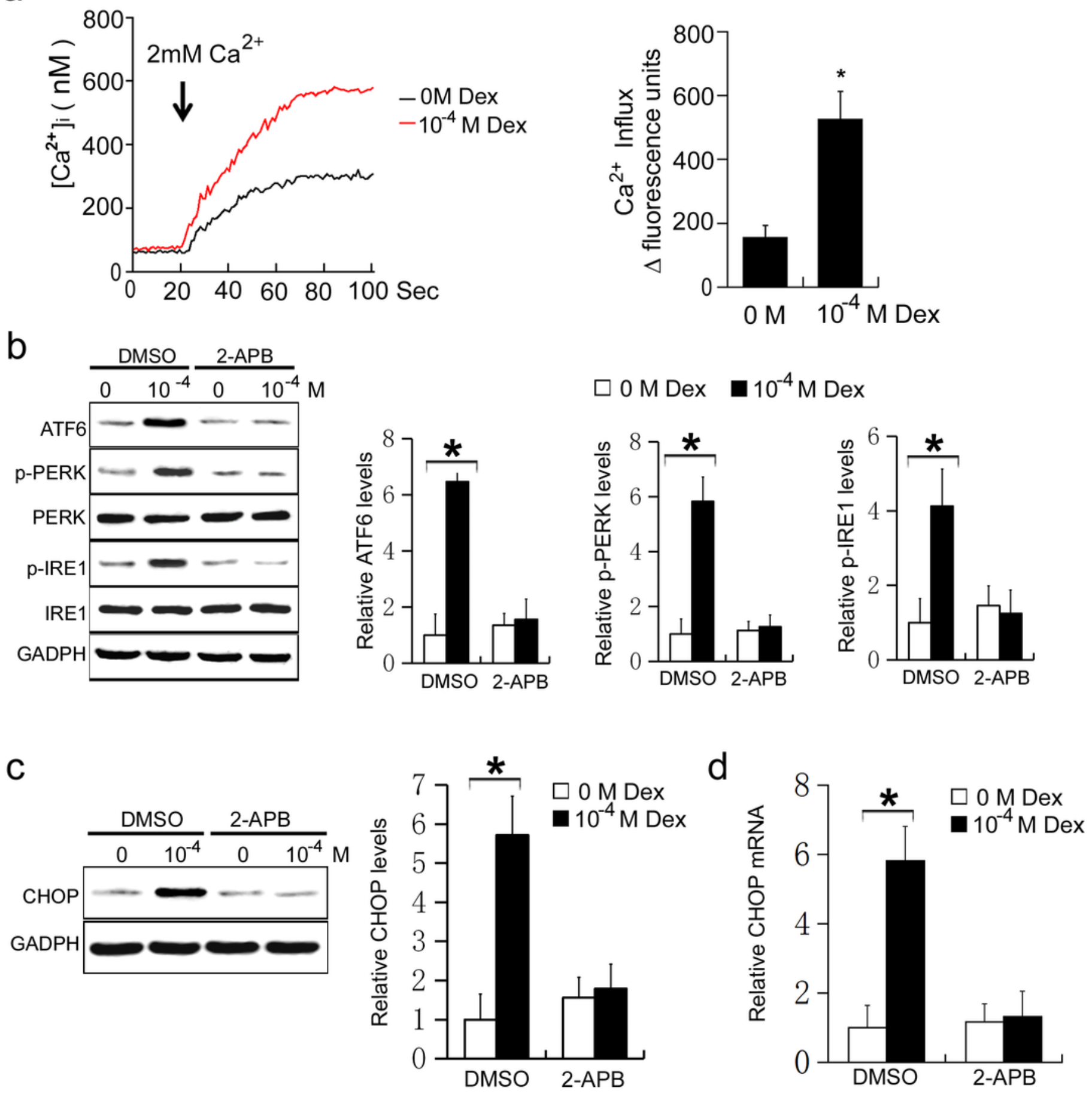

\section{Figure 3}

a. MC3T3-E1 cells were treated with 0 or 10-4 M Dex for $24 \mathrm{~h}$, and the calcium ion influx was detected by Fluo8-AM staining. MC3T3-E1 cells were treated with DMSO or 2-APB, and then treated with 0 or 10-4 M Dex for 24 h. b. Western blot of ATF6, phosphorylated PERK (p-PERK), PERK, phosphorylated IRE1 ( $p$ IRE1), and IRE1 in MC3T3-E1 cells. c. Western blot of CHOP in MC3T3-E1 cells. d. Real-time PCR of CHOP mRNA in MC3T3-E1 cells. 
Figure 4

a
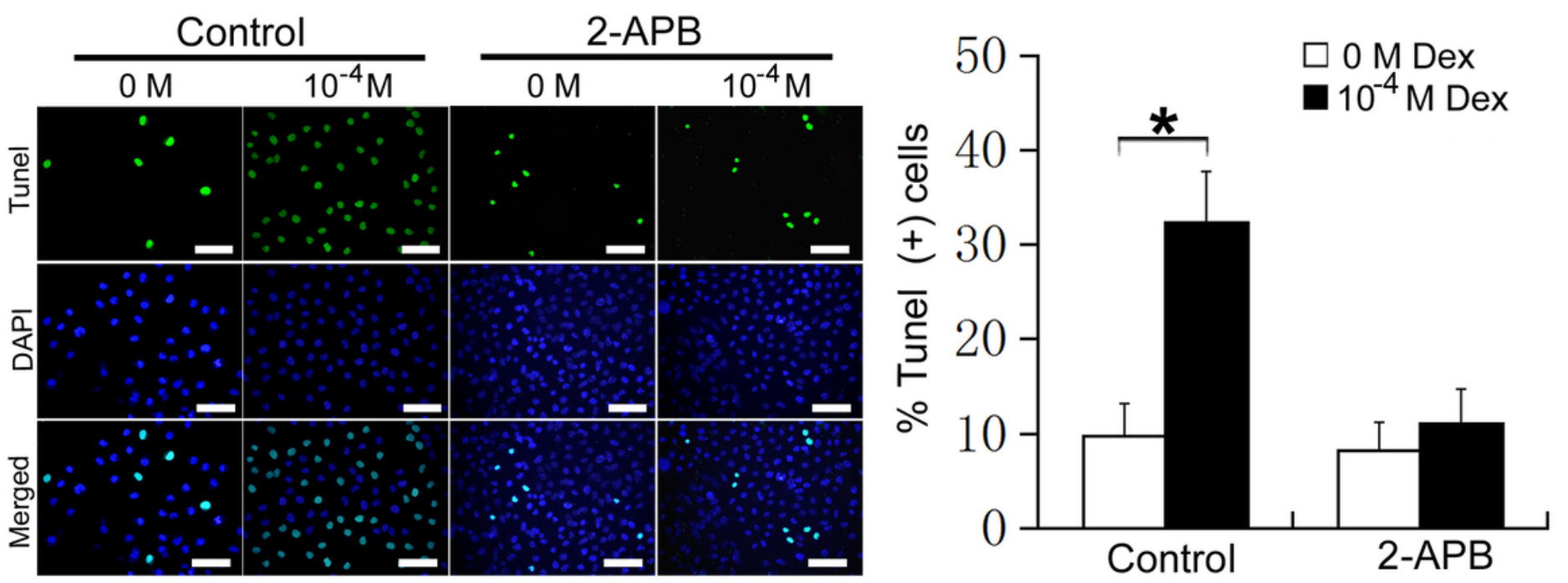

b

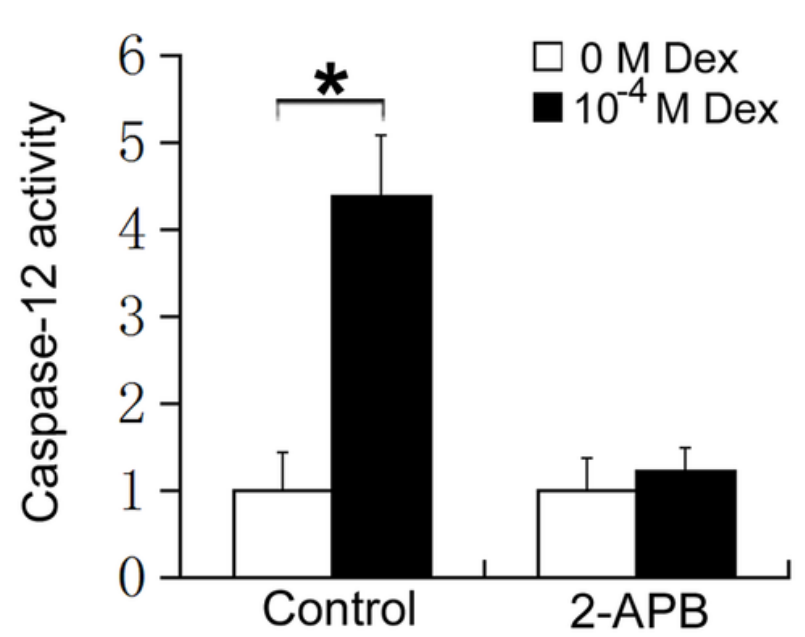

C

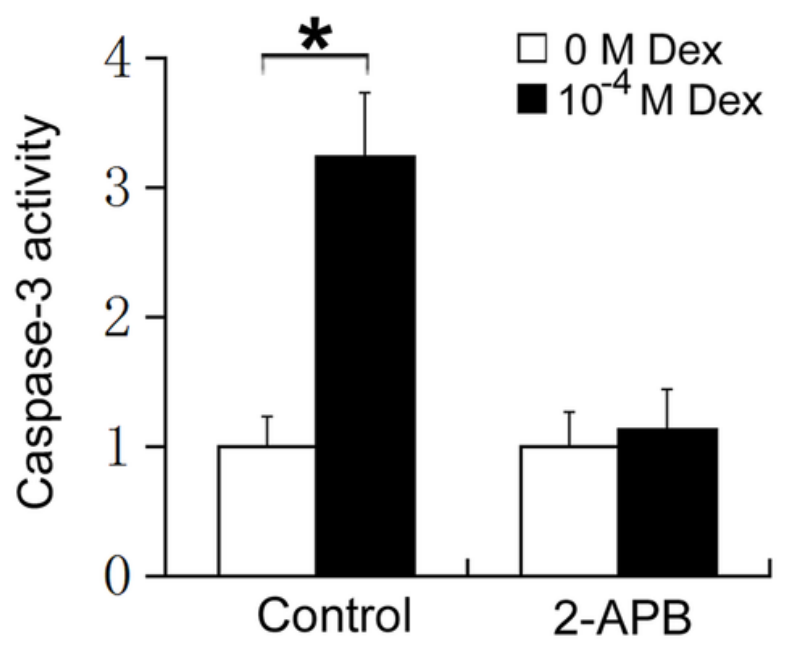

Figure 4

MC3T3-E1 cells were treated with DMSO or 2-APB, and then treated with 0 or 10-4 M Dex for $24 \mathrm{~h}$. a. TUNEL staining of MC3T3-E1 cells (Scale $=50 \mu \mathrm{m})$; b. Caspase-12 activity; c. Caspase-3 activity. 